\title{
Interval-Valued Intuitionistic Fuzzy Inference System for Supporting Corporate Financial Decisions
}

\author{
Petr Hajek, Vladimír Olej \\ Department of System Engineering and Informatics \\ Faculty of Economics and Administration, University of Pardubice \\ Pardubice, Czech Republic \\ Petr.Hajek,Vladimir.Olej@upce.cz
}

\begin{abstract}
Representing the inherent uncertainty in the corporate financial environment is critical for effective decisionmaking in this domain. This is attributed to the increasing complexity of such an environment. One way in which to address this issue is to represent financial attributes in terms of intervalvalued intuitionistic fuzzy sets. In this paper, a novel intervalvalued intuitionistic fuzzy inference system (IVIFIS) of the Takagi-Sugeno-Kang type is proposed. To calculate the output of the IVIFIS system, a defuzzification method is developed based on the weighted average of the consequents of if-then rules. To adapt the consequent parameters of the IVIFIS, a gradient algorithm is used. Then, by using two regression problems from the corporate financial domain, the dominance of the system over other state-of-the-art extensions of fuzzy inference systems is experimentally shown.
\end{abstract}

Keywords-interval-valued intuitionistic fuzzy sets; intervalvalued intuitionistic fuzzy inference system; defuzzification; financial decision

\section{INTRODUCTION}

To provide additional flexibility when expressing decision hesitancy and determinacy, Atanassov and Gargov [1] extended intuitionistic fuzzy sets (IFSs) [2] to interval-valued intuitionistic fuzzy sets (IVIFSs). The basic properties of IVIFSs and their operators were introduced by [3]. In fact, IVIFSs represent an extension of IFSs and interval-valued fuzzy sets (also known as grey sets). They are characterized by an interval-valued membership function and interval-valued non-membership function whose values are intervals rather than real numbers. This may be useful in situations where the inherent uncertainty of decision-makers is present and, thus, interval values are used to express preferences and nonpreferences. Therefore, IVIFSs have become a vital topic of interest in the decision-making domain [4]. Financial decisionmaking has represented a particularly important problem studied in the interval-valued intuitionistic fuzzy environment $[5]$.

Despite growing interest in IVIFSs, the corresponding extensions of fuzzy inference systems (FISs) have only recently been studied [6], [7]. It has been demonstrated that introducing an additional level of fuzziness through nonmembership functions and intuitionistic fuzzy indices into interval-valued FISs provides a more accurate regression performance over traditional FISs and interval-valued FISs [7].
However, the interval-valued intuitionistic fuzzy inference systems (IVIFISs) developed previously suffer from computational inefficiency owing to the composition of two subsystems, one for the membership function and the other for the non-membership function. Thus, the number of if-then rules is twice as large as that of traditional FISs. Moreover, the value of an additional design factor must be determined to assign weights to each subsystem. To overcome these problems, a more effective variant of IVIFISs is proposed in this study, with defuzzification performed directly on the consequents of interval-valued intuitionistic fuzzy if-then rules. In other words, the proposed IVIFIS contains only one set of consequent parameters, corresponding to the number of if-then rules. To achieve high computational efficiency, the IVIFIS is adapted by using a gradient algorithm previously successfully applied to adapt FISs [8] and their intuitionistic [9] and interval-valued intuitionistic extensions [7].

The remainder of this paper is organized as follows. In section II, the proposed IVIFIS of the Takagi-Sugeno-Kang (TSK) type is outlined and the process of its adaptation. In section III, the datasets used for the empirical experiments are introduced. Section IV shows the results of the experiments and comparative analysis with several state-of-the-art extensions of FISs. Finally, section V concludes the paper with a discussion and future directions.

\section{INTERVAL-VALUED INTUITIONISTIC FUZZY INFERENCE SYSTEM OF THE TSK TYPE}

For the sake of clarity, IVIFSs is first defined in this section. Next, this section presents each component of the proposed IVIFIS of the TSK type, namely fuzzification, inference in if-then rules and defuzzification (Fig. 1 provides an overview flowchart). Finally, the process of the adaptation of the IVIFIS consequent parameters is shown.

\section{A. Interval-Valued Intuitionistic Fuzzy Sets}

For a set $X$, an IVIFS $A$ is an object having the form [1]:

$$
A=\left\{\left\langle x, M_{A}(x), N_{A}(x)\right\rangle \mid x \in X\right\}
$$

where $M_{A}(x) \subset[0,1]$ and $N_{A}(x) \subset[0,1]$ represent the intervalvalued membership degree and interval-valued non- 
membership degree, respectively, $\sup \left(M_{A}(x)\right)+\sup \left(N_{A}(x)\right) \leq 1$, $\forall x \in X$.

Next, $M_{A}(x)$ and $N_{A}(x)$ can be defined as $M_{A}(x)=\left[M_{A L}(x)\right.$, $\left.M_{A U}(x)\right]$ and $N_{A}(x)=\left[N_{A L}(x), N_{A U}(x)\right]$, where $M_{A L}(x)$ and $M_{A U}(x)$ are the lower and upper extremes of the membership degree, and $N_{A L}(x)$ and $N_{A U}(x)$ are the lower and upper extremes of the non-membership degree, respectively. Hence, the IVIFS $A$ can be defined as $A=\left(\left[M_{A L}(x), M_{A U}(x)\right],\left[N_{A L}(x), N_{A U}(x)\right]\right)$. For each element $x \in X$, the hesitancy degree can be calculated as $\pi_{A}(x)=1-\left(M_{A}(x)+N_{A}(x)\right)$.

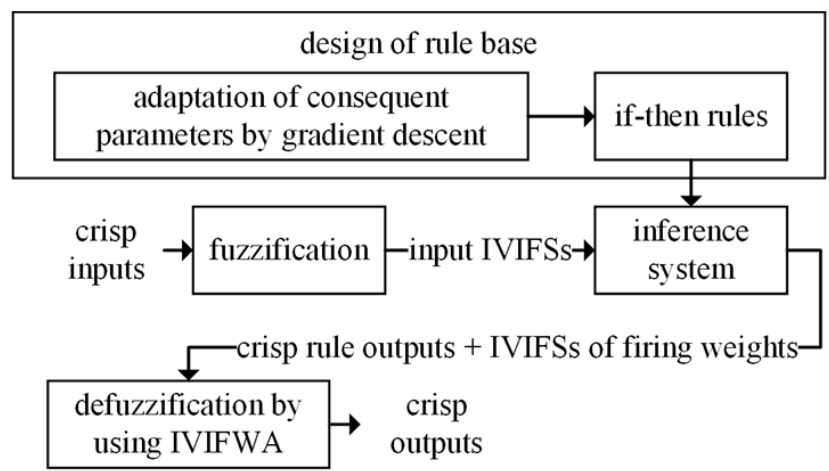

Fig. 1. Flowchart of the proposed IVIFIS.

\section{B. Fuzzification}

In the fuzzification process, the input attributes are compared with the interval-valued membership functions $M_{A}(x)$ and interval-valued non-membership functions $N_{A}(x)$. Here, these functions are represented by Gaussian functions as these require only two parameters to be determined:

$$
\begin{gathered}
M_{A L}(x)=\exp \left(-\left[(x-c)^{2} / 2 \sigma_{L}^{2}\right]\right), \\
M_{A U}(x)=\exp \left(-\left[(x-c)^{2} / 2 \sigma_{U}^{2}\right]\right), \\
N_{A L}(x)=1-\pi_{A}(x)-\exp \left(-\left[(x-c)^{2} / 2 \sigma_{U}^{2}\right]\right), \\
N_{A U}(x)=1-\pi_{A}(x)-\exp \left(-\left[(x-c)^{2} / 2 \sigma_{L}^{2}\right]\right),
\end{gathered}
$$

where $c$ denotes the centre of the IVIFS $A$, and $\sigma_{L}$ and $\sigma_{U}$ are the widths of the IVIFS $A$. The parameters $c$ and $\sigma$ for each input attribute can be determined automatically from the data using the subtractive clustering algorithm [10]. By adopting the approach used by [11] for interval-valued fuzzy membership functions, the parameters $\sigma_{L}$ and $\sigma_{U}$ were set as follows: $\sigma_{L}=$ $0.75 \times \sigma$ and $\sigma_{U}=1.25 \times \sigma$. Thus, the hesitancy of variance can be expressed in terms of interval values. Note that for the sake of simplicity, the hesitancy of the centres was not considered but this can be easily included into the model, see [7], [9]. Similarly, the value of the hesitancy degree was set as $\pi_{A}(x)=\pi_{A L}(x)=\pi_{A U}(x)=0$.

\section{Inference in If-Then Rules}

In the IVIFIS of a first-order TSK, the $k$-th if-then rule can be defined as follows:
$R_{k}$ : if $x_{1}$ is $A_{1, k}$ AND $x_{2}$ is $A_{2, k}$ AND ... AND $x_{i}$ is $A_{i, k}$ AND ...

AND $x_{n}$ is $A_{n, k}$ then $y_{k}=a_{0, k}+a_{1, k} x_{1}+\ldots+a_{i, k} x_{i}+\ldots+a_{n, k} x_{n}$,

where $A_{i, k}$ is the IVIFS for the $i$-th input attribute $x_{i}$ and $k$-th rule $R_{k}, y_{k}$ is the output of the $k$-th rule $R_{k}$, and $a_{0, k}, a_{1, k}, \ldots, a_{n, k}$ are the consequent parameters. To obtain the firing weight of each if-then rule, Gödel $t$-norm operators are applied as follows:

$$
\begin{aligned}
w_{k}^{M L} & =\min \left(M_{A_{1, k} L}\left(x_{1}\right), M_{A_{2, k} L}\left(x_{2}\right), \ldots, M_{A_{n, k} L}\left(x_{n}\right)\right), \\
w_{k}^{M U} & =\min \left(M_{A_{1, k} U}\left(x_{1}\right), M_{A_{2, k} U}\left(x_{2}\right), \ldots, M_{A_{n, k} U}\left(x_{n}\right)\right), \\
w_{k}^{N L} & =\max \left(N_{A_{1, k} L}\left(x_{1}\right), N_{A_{2, k} L}\left(x_{2}\right), \ldots, N_{A_{n, k} L}\left(x_{n}\right)\right), \\
w_{k}^{N U} & =\max \left(N_{A_{1, k} U}\left(x_{1}\right), N_{A_{2, k} U}\left(x_{2}\right), \ldots, N_{A_{n, k} U}\left(x_{n}\right)\right),
\end{aligned}
$$

where $w_{k}^{M L}$ and $w_{k}^{M U}$ are the lower and upper extremes of the membership degrees of firing weights and $w_{k}^{N L}$ and $w_{k}^{N U}$ are the lower and upper extremes of the non-membership degrees of firing weights, respectively.

\section{Defuzzification}

In the defuzzification process, the output of the IVIFIS is calculated as the weighted average of the outputs of $N$ if-then rules $R_{1}, R_{2}, \ldots, R_{k}, \ldots, R_{N}$. Since the subtractive clustering algorithm was used to estimate the premise parameters in the if-then rules, the number of if-then rules $N$ corresponds to the number of interval-valued membership (and non-membership) functions. To the best of our knowledge, no defuzzification methods have thus far been developed for IVIFISs. Therefore, here we propose an interval-valued intuitionistic fuzzy weighted average (IVIFWA) defuzzification method combining the centre of area (COA) method used for intuitionistic FISs [12] and the average defuzzification method defined for interval-valued FISs [13].

The IVIFWA is defined as follows:

$$
y_{\text {IVIFWA }}=\frac{\sum_{k=1}^{N} y_{k} \frac{\left(w_{k}^{M L}-w_{k}^{N L}\right)+\left(w_{k}^{M U}-w_{k}^{N U}\right)}{2}}{\sum_{k=1}^{N} \frac{\left(w_{k}^{M L}-w_{k}^{N L}\right)+\left(w_{k}^{M U}-w_{k}^{N U}\right)}{2}} .
$$

The output $y_{\text {IVIFWA }}$ is calculated from the positive differences between the firing weights $w_{k}{ }^{M L}$ and $w_{k}{ }^{N L}$, on the one hand, and between $w_{k}^{M U}$ and $w_{k}{ }^{N U}$, on the other hand. In fact, the if-then rules with these two positive differences represent all outputs with the lower and upper degree of acceptance higher than the lower and upper degree of nonacceptance, $w_{k}^{M L}>w_{k}{ }^{N L}$ and $w_{k}^{M U}>w_{k}{ }^{N U}$. Note that if $w_{k}{ }^{M L}=$ $w_{k}^{M U}$ and $w_{k}^{N L}=w_{k}^{N U}$, the COA method defined for intuitionistic FISs is obtained.

\section{Numerical Example}

Let $x_{1}=0.5$ and $x_{2}=1$ be the values of input attributes, and $R_{1}$ and $R_{2}$ be if-then rules defined as follows: 


$$
\begin{aligned}
& R_{1}: \text { if } x_{1} \text { is } A_{1,1} \text { AND } x_{2} \text { is } A_{2,1} \text { then } y_{1}=0.1+0.2 x_{1}+0.5 x_{2}, \\
& R_{2}: \text { if } x_{1} \text { is } A_{1,2} \text { AND } x_{2} \text { is } A_{2,2} \text { then } y_{2}=0.5+0.1 x_{1}+0.3 x_{2},
\end{aligned}
$$

where $\quad A_{1,1}=([0.7,0.8],[0.1,0.2]), \quad A_{2,1}=([0.5,0.7],[0.0,0.3])$, $A_{1,2}=([0.6,0.9],[0.0,0.1])$, and $A_{2,2}=([0.6,0.8],[0.1,0.2])$. Then, the firing weights of the if-then rules are calculated using eq. (7)-(10) as $w_{1}=([0.5,0.7],[0.1,0.3])$ and $w_{2}=([0.6,0.8],[0.1,0.2])$. For instance, $w_{1}{ }^{M L}=\min (0.7,0.5)$ and $w_{1}{ }^{N L}=\max (0.1,0.0)$. Finally, the output $y_{\text {IVIFWA }}$ of the IVIFIS is obtained as:

$$
y_{\mathrm{IVIFWA}}=(0.7 \times 0.4+0.85 \times 0.55) /(0.4+0.55)=0.787 \text {. }
$$

\section{E. Adaptation of the Consequent Parameters}

As noted above, the premise parameters in the IVIFIS were set based on the subtractive clustering algorithm. In the algorithm, the radius of influence is the parameter responsible for the calculation of both the number of interval-valued membership (and non-membership) functions and the number of if-then rules. As a result, what remains to be adapted are the consequent parameters $a_{0, k}, a_{1, k}, \ldots, a_{n, k}$. Here, the gradient descent algorithm is used to adapt these parameters because it has been effective in the adaptation of FISs [8], intuitionistic FISs [9] and the interval type-2 Atanassov-intuitionistic fuzzy logic system (IT2AIFLS) [7].

Let $E=\Sigma E_{i}$ be the total error, $E_{i}=\left(y_{i}-y_{i}^{\prime}\right)^{2}$, where $y_{i}$ is the target and $y_{i}^{\prime}$ is the predicted output for the $i$-th input vector. Then, the gradient descent algorithm can be defined as:

$$
\alpha(t+1)=\alpha(t)-\eta \frac{\partial E}{\partial \alpha},
$$

where $\alpha$ is a generic parameter and $\eta$ denotes the learning rate. The number of consequent parameters to be adapted can be calculated as $(n+1) \times k$. Note that this is two times less than that for the IT2AIFLS, where these parameters have to be separately updated for the membership function and nonmembership function subsystems.

\section{F. IVIFIS Overview}

The process of IVIFIS generation and adaptation can be summarized as follows:

Step 1. Generate the centres of Gaussian interval-valued membership functions $M_{A}(x)$ and interval-valued nonmembership functions $N_{A}(x)$ using the subtractive clustering algorithm and set $\sigma_{L}=0.75 \times \sigma$ and $\sigma_{U}=1.25 \times \sigma$. $\pi_{A}(x)=\pi_{A L}(x)=\pi_{A U}(x)=0$.

Step 2. Fix the premise parameters of if-then rules as generated by the subtractive clustering algorithm and set the corresponding values of consequent parameters as initial values.

Step 3. Apply the Gödel $t$-norm operators to calculate the firing weights of the if-then rules and use the IVIFWA defuzzification method to calculate the IVIFIS output.
Step 4. Calculate the total error between the IVIFIS output and target output.

Step 5. Adapt the consequent parameters using the gradient descent algorithm to minimize the total error.

\section{DATA FOR CORPORATE FINANCIAL PREDICTIONS}

In this study, the IVIFIS is applied to two corporate financial decision-making problems: capital structure prediction [14] and profitability prediction [15]. Both problems have received considerable attention in empirical financial research. However, no one, to the best of our knowledge, has considered the intrinsic and inherent uncertainty present in these decision-making problems. Indeed, it has been shown that financial decision-makers use interval values to express preferences and non-preferences rather than exact numbers [5]. Moreover, noise in the financial data (e.g., the application of different accounting principles), the often contradictory financial objectives of the stakeholders and linguistic uncertainties are associated with corporate financial decisions [16]. This makes the problems even more difficult to be modelled by using traditional linear regression methods.

Here, the IVIFIS is used to model the effect of the most important decision-making factors on future capital structure and profitability. Capital structure is usually quantified by using a leverage ratio, while profitability ratios represent corporate profitability. In agreement with the literature [14], [15], total debt to total assets (TD/TA) is used as the leverage ratio and return on assets (ROA) as the profitability ratio. Previous empirical studies generally agree that leverage increases with firm size and dividend payments and decreases with profit and the market-to-book ratio (PBV) [14]. Similarly, profitability is mainly driven by the following determinants [15]: firm size, dividend yield, dividend payments and marketto-book ratio. In this study, the common measures of these indictors were used, namely TA to represent firm size, the payout ratio (PR) for dividend payments and operating income (OI) for profit, dividend yield (DY) and the PBV. In addition, recent empirical evidence suggests that the tone and content of managerial comments in corporate annual reports can be used as indicators of future financial decisions [17]. Specifically, a net positive sentiment (NPS) indicates higher profitability (and lower leverage), while comments focused on profitability (PROF) and leverage (LEV) suggest better future performance with respect to their corresponding financial indicators [18]. To take into account the delay in the effects of the abovementioned indicators, the prediction models for capital structure and profitability, respectively, were designed as follows:

$$
\begin{aligned}
\mathrm{TD} / \mathrm{TA}^{t+2} & =f\left(\mathrm{TA}^{t}, \mathrm{PR}^{t}, \mathrm{OI}^{t}, \mathrm{PBV}^{t}, \mathrm{NPS}^{t}, \mathrm{PROF}^{t}, \mathrm{LEV}^{t}\right), \\
\mathrm{ROA}^{t+2} & =f\left(\mathrm{TA}^{t}, \mathrm{PR}^{t}, \mathrm{DY}^{t}, \mathrm{PBV}^{t}, \mathrm{NPS}^{t}, \mathrm{PROF}^{t}, \mathrm{LEV}^{t}\right),
\end{aligned}
$$

where $t$ represents the year. The data were collected for 1380 US firms from the Value Line database in 2013 (input variables) and 2015 (output variables). The selection of the firms was based on the following criteria: (1) the firms had to be listed on one of the major US stock exchanges, the Nasdaq 
or New York Stock Exchange (NYSE), and (2) market capitalization of $>100$ mil. USD was required to minimize the impact of risk factors related particularly to small firms. The financial indicators were collected from the Value Line database for these firms, while managerial comments were obtained from the annual reports (item 7: management's discussion and analysis) downloaded from the Edgar system (www.sec.gov/ edgar.shtml). For the NPS, the word lists (positive and negative) specifically developed for the financial domain were used, available at www3.nd.edu/ mcdonald/ Word_Lists.html. In agreement with previous related studies [18], the NPS was calculated as (pos-neg)/(pos+neg), where pos and neg are the counts of positive and negative words (related to the length of the text documents), respectively. Similarly, the word lists for leverage and profitability topics were used to calculate the relative frequencies representing the LEV and PROF variables. Statistica 12 (Text Mining tool) was used to obtain these frequencies. The basic descriptive statistics of the data are presented in Table I. Regarding the output variables, $\mathrm{TD} / \mathrm{TA}^{2015}$ ranges from 0 to 1 , while $\mathrm{ROA}^{2015}$ ranges from -2.5 to 12.0 .

TABLE I. DESCRIPTIVE STATISTICS OF THE VARIABLES

\begin{tabular}{|c|c|c|c|}
\hline Var. & Mean \pm St.Dev. & Var. & Mean \pm St.Dev. \\
\hline $\mathrm{TA}^{2013}$ & $15,308 \pm 49,602$ & $\mathrm{NPS}^{2013}$ & $-0.342 \pm 0.098$ \\
\hline $\mathrm{PR}^{2013}$ & $0.479 \pm 2.820$ & $\mathrm{PROF}^{2013}$ & $0.007 \pm 0.002$ \\
\hline $\mathrm{OI}^{2013}$ & $782 \pm 2,743$ & $\mathrm{LEV}^{2013}$ & $0.030 \pm 0.005$ \\
\hline $\mathrm{PBV}^{2013}$ & $6.34 \pm 53.45$ & $\mathrm{TD} / \mathrm{TA}^{2015}$ & $0.481 \pm 0.197$ \\
\hline $\mathrm{DY}^{2013}$ & $0.014 \pm 0.023$ & $\mathrm{ROA}^{2015}$ & $0.219 \pm 0.592$ \\
\hline
\end{tabular}

\section{EXPERIMENTAL RESULTS}

Training/testing data were generated by using $5 \times 2$ crossvalidation: this two-fold cross-validation was repeated five times. Prediction quality was measured by the RMSE of the testing data. All the experiments were conducted by using the Matlab Fuzzy Logic Toolbox.

First, the premise parameters of the IVIFIS were obtained by using the subtractive clustering algorithm. In the experiments, the radius of influence was set to avoid underand over-fitting. The value of the radius was set to change the numbers of cluster centres, which are equal to the numbers of interval-valued membership (and non-membership) functions and the numbers of if-then rules $N$. Specifically, three settings were tested, $N=\{2,3,5\}$. The consequent parameters of the ifthen rules were adapted by using the gradient descent algorithm with the maximum number of epochs equal to 500 and the learning rate $\eta=0.01$.

Fig. 2 shows that the IVIFIS with $N=3$ if-then rules (and interval-valued membership and non-membership functions) achieved the best results for both prediction problems. Therefore, this setting was fixed in the further experiments. Obviously, there was no need to increase the number of cluster centres above five, as this would only result in an overfitted IVIFIS. Moreover, the IVIFIS with three rules (and labels) is also preferable in terms of interpretability at both the rule base level and the fuzzy partition level. For the example of the NPS variable, the interval-valued membership and nonmembership functions are depicted in Fig. 3 and Fig. 4, respectively. The if-then rule $R_{k}, k=1,2,3$, for the capital structure prediction and profitability prediction, respectively, can be defined as follows:

\section{Capital structure prediction:}

$R_{k}$ : if $\mathrm{TA}^{2013}$ is $A_{1, k}$ AND $\mathrm{PR}^{2013}$ is $A_{2, k}$ AND OI ${ }^{2013}$ is $A_{3, k}$ AND PBV 2013 is $A_{4, k}$ AND NPS ${ }^{2013}$ is $A_{5, k}$ AND PROF $^{2013}$ is $A_{6, k}$ AND LEV 2013 is $A_{7, k}$ then TD/TA ${ }_{k}^{2015}=a_{0,1}+a_{1, k} \mathrm{TA}^{2013+}$ $a_{2, k} \mathrm{PR}^{2013}+a_{3, k} \mathrm{OI}^{2013}+a_{4, k} \mathrm{PBV}^{2013}+a_{5, k} \mathrm{NPS}^{2013}+a_{6, k} \mathrm{PROF}^{2013}+$ $a_{7, k} \mathrm{LEV}^{2013}$,

\section{Profitability prediction:}

$R_{k}$ : if TA ${ }^{2013}$ is $A_{1, k}$ AND PR ${ }^{2013}$ is $A_{2, k}$ AND DY 2013 is $A_{3, k}$ AND PBV 2013 is $A_{4, k}$ AND NPS $^{2013}$ is $A_{5, k}$ AND PROF 2013 is $A_{6, k}$ AND LEV 2013 is $A_{7, k}$ then $\mathrm{ROA}_{k}^{2015}=a_{0,1}+a_{1, k} \mathrm{TA}^{2013}$ $+a_{2, k} \mathrm{PR}^{2013}+a_{3, k} \mathrm{DY}^{2013}+a_{4, k} \mathrm{PBV}^{2013}+a_{5, k} \mathrm{NPS}^{2013}+a_{6, k} \mathrm{PROF}^{2013}$

$+a_{7, k} \mathrm{LEV}^{2013}$,
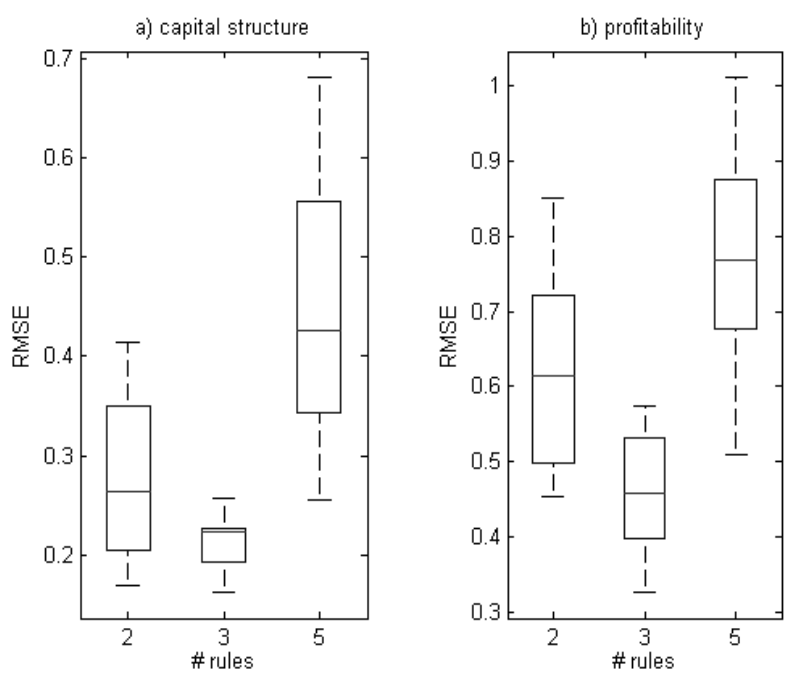

Fig. 2. RMSE of the IVIFIS for different numbers of rules.

To investigate the input-output relationships, the sensitivity of the outputs to each input was tested. While the effects of the financial indicators were linear and in agreement with the literature [14], [15], the effects of the tone and content of managerial comments were non-linear, as presented in Fig. 4 and Fig. 5 (average effects over all data partitions are depicted for the sake of clarity). Specifically, comments that are more positive indicate lower leverage to a certain point. On the contrary, too positive comments may indicate high leverage (Fig. 4). When focus is placed on leverage in the managerial comments, it generally indicates lower leverage. Similarly, higher profitability was associated with positive comments and an emphasis on profitability in the comments.

In the further experiments, the results of the IVIFIS were compared with the following FISs with $N=3$ if-then rules adapted by using the gradient descent algorithm with the same 
settings as for the IVIFIS: (1) ANFIS [8], (2) IT2AIFLS [7], (3) the intuitionistic FIS (IFIS) [9] and (4) the interval type-2 fuzzy logic system with the enhanced Karnik-Mendel algorithm (IT2FLS-EKM) [19]. These experiments were performed in Matlab R2013b. In addition, the performance of the IVIFIS was compared with the Wang-Mendel (WM) algorithm [20], a traditional algorithm used to adapt FISs, and with the following state-of-the-art evolutionary FISs: (1) an FIS learnt by using genetic programming grammar operators (GFS-GPG) [21] and (2) an FIS with genetic learning within MOGUL-TSK [22]. The implementations provided in the Keel 3.0 software environment were used. To obtain the comparable granularity of the FISs, the number of labels (membership functions) was set to three in WM, GFS-GPG and MOGULTSK. The default values were used for the remaining settings of the GFS-GPG parameters: $N=8$ if-then rules, a population size of 30 , a number of iterations of 10000 , a mutation probability of 0.01 and a migration probability of 0.001 . The MOGUL-TSK was trained with 100 evolutionary strategy iterations, 500 evolutionary strategy MOGUL iterations, 500 iterations of selection and 1000 iterations of tuning.
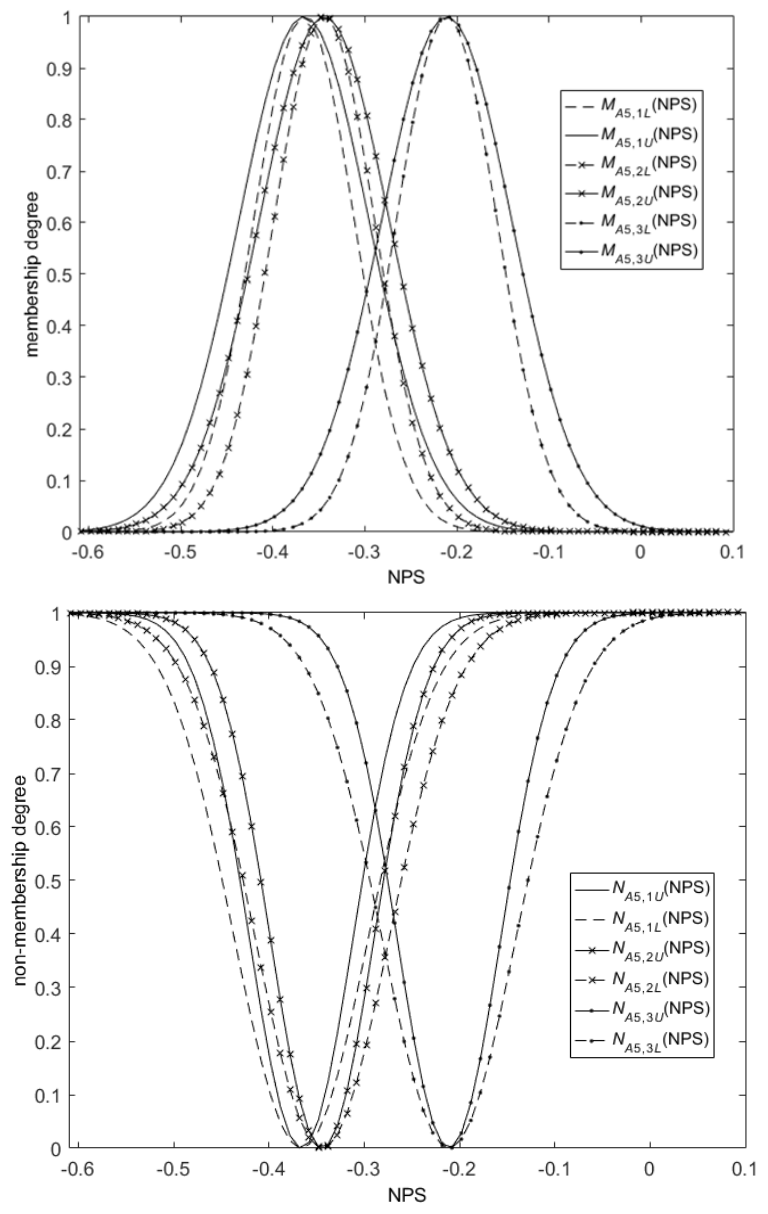

Fig. 3. An example of the interval-valued membership and non-membership functions for the NPS variable.
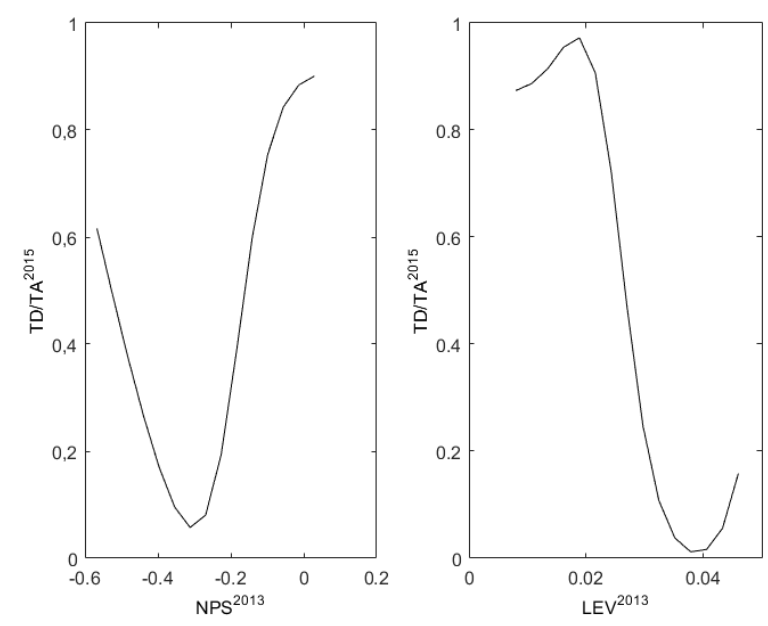

Fig. 4. The effect of NPS ${ }^{2013}$ and LEV 2013 on capital structure.
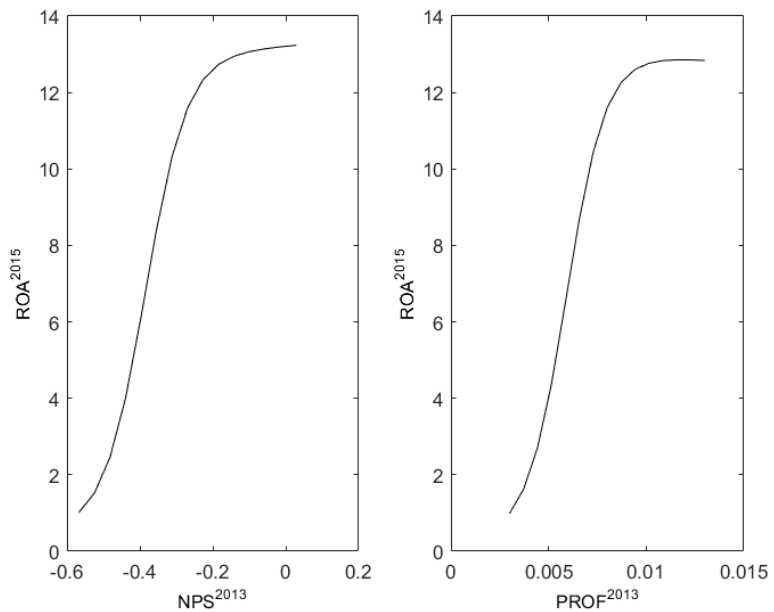

Fig. 5. The effect of NPS ${ }^{2013}$ and $\mathrm{PROF}^{2013}$ on profitability.

The results of the comparative experiments for the prediction of capital structure and profitability are presented in Table II and Table III, respectively. For the capital structure prediction problem, the WM algorithm performed best. Several methods provided statistically comparable results (using the paired Student's $t$-test at $p=0.5$ ), including the IT2AIFLS. However, for the WM and GFS-GPG, this was achieved at the cost of a higher number of if-then rules. From the set of FIS extensions, those working with interval-valued membership functions were more accurate compared with their intuitionistic counterparts, suggesting that uncertainty expressed in terms of intervals may be more effective for capital structure decisionmaking. For the problem of profitability prediction, GFS-GPG performed the best. Although the IVIFIS did not perform the best in terms of the RMSE, it provided both statistically similar prediction performance to GFS-GPG and highly interpretable results at the rule base level. Surprisingly, the WM algorithm performed worst due to over-fitting several data partitions. Considering the compared FIS generalizations, on the one hand, they performed significantly worse than the best prediction method but, on the other hand, the differences to the IVIFIS performance were not significant at $p=0.05$. 
TABLE II. COMPARISON OF THE RESULTS WITH ALTERNATIVE FISS CAPITAL STRUCTURE PREDICTION

\begin{tabular}{|c|c|c|}
\hline Method & RMSE (Mean \pm St.Dev.) & \# Rules (Mean) \\
\hline ANFIS [8] & $0.2684 \pm 0.2564$ & 3.0 \\
\hline IT2AIFLS [7] & $\mathbf{0 . 2 0 8 3} \pm \mathbf{0 . 0 2 8 4}$ & 3.0 \\
\hline IFIS [9] & $0.2593 \pm 0.1208$ & 3.0 \\
\hline IT2FLS-EKM [19] & $\mathbf{0 . 2 0 3 3} \pm \mathbf{0 . 1 1 6 9}$ & 3.0 \\
\hline WM [20] & $\mathbf{0 . 2 0 0 7} \pm \mathbf{0 . 0 4 3 0}$ & 30.8 \\
\hline GFS-GPG [21] & $\mathbf{0 . 2 0 2 2} \pm \mathbf{0 . 0 3 5 1}$ & 8.0 \\
\hline MOGUL-TSK [22] & $0.2869 \pm 0.2017$ & 23.4 \\
\hline IVIFIS & $\mathbf{0 . 2 0 2 9} \pm \mathbf{0 . 0 3 3 7}$ & 3.0 \\
\hline
\end{tabular}

TABLE III. COMPARISON OF THE RESULTS WITH ALTERNATIVE FISS PROFITABILITY PREDICTION

\begin{tabular}{|c|c|c|}
\hline Method & RMSE (Mean \pm St.Dev.) & \# Rules (Mean) \\
\hline ANFIS [8] & $0.5469 \pm 0.0971$ & 3.0 \\
\hline IT2AIFLS [7] & $0.5953 \pm 0.0812$ & 3.0 \\
\hline IFIS [9] & $0.6078 \pm 0.1036$ & 3.0 \\
\hline IT2FLS-EKM [19] & $0.5914 \pm 0.2178$ & 3.0 \\
\hline WM [20] & $2.4615 \pm 1.4149$ & 37.6 \\
\hline GFS-GPG [21] & $\mathbf{0 . 3 7 6 4} \pm \mathbf{0 . 3 4 9 3}$ & 8.0 \\
\hline MOGUL-TSK [22] & $\mathbf{0 . 4 6 2 9} \pm \mathbf{0 . 1 7 8 4}$ & 21.8 \\
\hline IVIFIS & $\mathbf{0 . 4 7 8 9} \pm \mathbf{0 . 1 0 7 5}$ & 3.0 \\
\hline
\end{tabular}

\section{CONCLUSION}

In this paper, an IVIFIS is proposed, representing a novel extension of FISs. The high computational efficiency of the proposed system is achieved through the defuzzification performed directly on the consequents of the if-then rules. Thus, the number of parameters to be adapted is decreased compared with the recently proposed IT2AIFLS. The effectiveness of the IVIFIS was demonstrated in the domain of financial decision-making, namely for capital structure and profitability prediction. It was shown that the proposed system can model complex input-output relationships in a highly uncertain financial environment. Indeed, the results suggest that introducing additional freedom with the design of membership (and non-membership) functions may produce highly accurate and interpretable IVIFISs.

Finally, a number of limitations of the present study need to be noted. First, the application of the gradient descent algorithm can be difficult for more complex and large-scale problems due to its slow convergence and susceptibility to a local minimum of error function. Therefore, further research might explore the adaptation of the IVIFIS by using evolutionary algorithms, in a similar manner as for IT2FLSs [23], [24]. Second, several premise parameters were fixed for the sake of simplicity, such as the hesitancy degrees of nonmembership functions and the widths of the IVIFSs. These can also be adapted in future studies to raise accuracy. Finally, other methods such as association rules [11], [25] and other evolutionary rule learning or selection [26], [27] can be used to generate membership and non-membership functions and better fit the variability of the patterns in the data.

\section{REFERENCES}

[1] K. Atanassov and G. Gargov, "Interval valued intuitionistic fuzzy sets," Fuzzy Sets Syst., vol. 31, no. 3, pp. 343-349, 1989.

[2] K. T. Atanassov, "Intuitionistic fuzzy sets," Fuzzy Sets Syst., vol. 20, no. 1, pp. 87-96, 1986.

[3] K. T. Atanassov, "Operators over interval valued intuitionistic fuzzy sets," Fuzzy Sets Syst., vol. 64, no. 2, pp. 159-174, 1994.

[4] T. Y. Chen, "A prioritized aggregation operator-based approach to multiple criteria decision making using interval-valued intuitionistic fuzzy sets: A comparative perspective," Inf. Sci. (Ny)., vol. 281, pp. 97112, 2014.

[5] Z. Xu and M. Xia, "Induced generalized intuitionistic fuzzy operators," Knowledge-Based Syst., vol. 24, no. 2, pp. 197-209, 2011.

[6] I. Eyoh, R. John, and G. De Maere, "Interval type-2 intuitionistic fuzzy logic system for non-linear system prediction," in 2016 IEEE International Conference on Systems, Man, and Cybernetics, SMC 2016 - Conference Proceedings, 2017, pp. 1063-1068.

[7] I. Eyoh, R. John, and G. De Maere, "Interval type-2 intuitionistic fuzzy logic for regression problems," IEEE Trans. Fuzzy Syst., vol. PP, no. 99, pp. 1-14, 2017.

[8] J. S. R. Jang, "ANFIS: Adaptive-network-based fuzzy inference system," IEEE Trans. Syst. Man Cybern., vol. 23, no. 3, pp. 665-685, 1993.

[9] P. Hajek and V. Olej, "Intuitionistic neuro-fuzzy network with evolutionary adaptation," Evol. Syst., vol. 8, no. 1, pp. 35-47, 2017.

[10] S. L. Chiu, "Fuzzy model identification based on cluster estimation," $J$. Intell. Fuzzy Syst., vol. 2, no. 3, pp. 267-278, 1994.

[11] J. A. Sanz, A. Fernandez, H. Bustince, and F. Herrera, "IVTURS: A linguistic fuzzy rule-based classification system based on a new intervalvalued fuzzy reasoning method with tuning and rule selection," IEEE Trans. Fuzzy Syst., vol. 21, no. 3, pp. 399-411, 2013.

[12] P. Hajek and V. Olej, "Defuzzification methods in intuitionistic fuzzy inference systems of Takagi-Sugeno type: The case of corporate bankruptcy prediction," in 2014 11th International Conference on Fuzzy Systems and Knowledge Discovery, FSKD 2014, 2014, pp. 232-236.

[13] Q. Liang and J. M. Mendel, "Interval type-2 fuzzy logic systems: theory and design," IEEE Trans. Fuzzy Syst., vol. 8, no. 5, pp. 535-550, 2000.

[14] M. Z. Frank and V. K. Goyal, "Capital factors structure decisions: Which are reliably important?," Financ. Manag., vol. 38, no. 1, pp. 137, 2009.

[15] E. F. Fama and K. R. French, "Forecasting profitability and earnings," $J$. Bus., vol. 73, no. 2, pp. 161-175, 2000.

[16] P. Hajek and O. Prochazka, "Interval-valued fuzzy cognitive maps for supporting business decisions," in IEEE International Conference on Fuzzy Systems (FUZZ-IEEE), 2016, pp. 531-536.

[17] P. Hajek, V. Olej, and R. Myskova, "Forecasting corporate financial performance using sentiment in annual reports for stakeholders' decision-making," Technol. Econ. Dev. Econ., vol. 20, pp. 721-738, 2014.

[18] R. Myskova and P. Hajek, "Comprehensive assessment of firm financial performance using financial ratios and linguistic analysis of annual reports," J. Int. Stud., vol. 10, no. 4, pp. 96-108, 2017.

[19] D. Wu and J. M. Mendel, "Enhanced Karnik-Mendel algorithms," IEEE Trans. Fuzzy Syst., vol. 17, no. 4, pp. 923-934, 2009.

[20] L. X. Wang and J. M. Mendel, "Generating fuzzy rules by learning from examples," IEEE Trans. Syst. Man Cybern., vol. 22, no. 6, pp. 1414 $1427,1992$.

[21] L. Sànchez, I. Couso, and J. A. Corrales, "Combining GP operators with SA search to evolve fuzzy rule based classifiers," Inf. Sci. (Ny)., vol. 136, no. 1-4, pp. 175-191, 2001. 
[22] R. Alcalá, J. Alcalá-Fdez, J. Casillas, O. Cordón, and F. Herrera, "Local identification of prototypes for genetic learning of accurate TSK fuzzy rule-based systems," Int. J. Intell. Syst., vol. 22, no. 9, pp. 909-941, 2007.

[23] M. Almaraashi, R. John, A. Hopgood, and S. Ahmadi, "Learning of interval and general type-2 fuzzy logic systems using simulated annealing: Theory and practice," Inf. Sci. (Ny)., vol. 360, pp. 21-42, 2016.

[24] O. Castillo, R. Martínez-Marroquín, P. Melin, F. Valdez, and J. Soria, "Comparative study of bio-inspired algorithms applied to the optimization of type-1 and type-2 fuzzy controllers for an autonomous mobile robot," Inf. Sci. (Ny)., vol. 192, pp. 19-38, 2012.

[25] P. Hajek, "Predicting corporate investment/non-investment grade by using interval-valued fuzzy rule-based systems - A cross-region analysis," Appl. Soft Comput. J., vol. 62, pp. 73-85, 2018.

[26] O. Cordón, "A historical review of evolutionary learning methods for Mamdani-type fuzzy rule-based systems: Designing interpretable genetic fuzzy systems," International Journal of Approximate Reasoning, vol. 52, no. 6. pp. 894-913, 2011.

[27] F. Herrera, "Genetic fuzzy systems: Taxonomy, current research trends and prospects," Evol. Intell., vol. 1, no. 1, pp. 27-46, 2008. 